\title{
THE ORIENTATION OF THE GRAINS IN A DRIED PHOTOGRAPHIC EMULSION*
}

\author{
BY \\ L. SilbERSTEIN
}

In my first paper on this subject the number of grains (small plates) whose inclination in the initial state falls within the limits $\theta$ and $\theta+d \theta$ was, through an obvious inadvertency, assumed to be $\frac{2}{\pi} N d \theta$, as if the plate-normals were confined to a plane. The directions of these normals being haphazardly distributed in space, the number in question is manifestly equal $N$ times the ratio of the area of the spherical zone $d \theta$ to that of the hemisphere, i.e., $N \sin \theta \mathrm{d} \theta \ldots \ldots \ldots \ldots \ldots \ldots \ldots(a)$

This then has to replace the first (and only) expression on p. 171.

The remainder of p. 171, as well as the whole of p. 172 and p. 173 up to section 1 are unaffected, while the formulae concerning the orientation in the final, dry state of the emulsion are to be modified as follows:-

1. By the "sine-law," $\sin \theta^{\prime}=k \sin \theta$, formula (1), we have, as before, $\cos \theta^{\prime}=\sqrt{1-k^{2} \sin ^{2} \theta}$, and therefore, by $(a)$, for the efficiency coefficient $\kappa$, as defined on p. 171,

or, putting $\cos \theta=u$,

$$
\kappa=\int_{0}^{\pi / 2} \sin \theta \sqrt{1-k^{2} \sin ^{2} \theta} \mathrm{d} \theta
$$

$$
\kappa=\int_{0}^{1} \sqrt{1-k^{2}+k^{2} u^{2}} \mathrm{~d} u .
$$

The integral is easily evaluated and gives the efficiency coefficient

$$
\kappa=\frac{1}{2}+\frac{1-k^{2}}{4 k} \log \frac{1+k}{1-k}
$$

or, developing the log,

$$
\kappa=1-\frac{k^{2}}{1.3}-\frac{k^{4}}{3.5}-\frac{k^{6}}{5.7}-
$$

* Corrigenda to a paper of equal title published in the March number of this Journal, pp. 171-177, 1921. 
of which, the contraction ratio $k$ being a small fraction, the first two terms will suffice in practice. For $k=0$ we have $k=1$, an obvious result, and for $k=1$ (initial state) $\kappa=1 / 2$, as it should be. The whole range of $\kappa$ is thus contained between $1 / 2$ and 1 .

If the thickness $\epsilon a$ of the grain-plates is taken into account (p. 174), the efficient area is increased by $a \epsilon k \cdot \sin ^{2} \theta=1 / 2 a \epsilon k$, and the correction term $\frac{1}{2} \epsilon k$ is to be added to the value of $\kappa$ given above.

2. By the alternative assumption on the contraction process, the "tangent-law," $\tan \theta^{\prime}=k \tan \theta$, we have $\cos ^{2} \theta^{\prime}=1:\left(1+k^{2} \tan ^{2} \theta\right)$, as before, and, by (a), substituting $u=\sin ^{2} \theta$,

$$
\kappa=\int_{0}^{\pi / 2} \frac{\sin \theta \cos \theta \mathrm{d} \theta}{\sqrt{1-\left(1-k^{2}\right) \sin ^{2} \theta}}=1 / 2 \int_{0}^{1} \frac{\mathrm{d} u}{\sqrt{1-\left(1-k^{2}\right) u}} .
$$

Thus the efficiency coefficient corresponding to the tangent-law is simply

$$
\kappa=\frac{1}{1+k} .
$$

This gives for $k=1$ and $k=0$ the values $k=\frac{1}{2}$ and 1 , as above, while for intermediate $k$ the values of $k$ are smaller than those given by (I). For contractions such as $k=\frac{1}{10}$ the two $k$-values differ only by about 9 percent. The following table (replacing that of p. 175) gives $k$ on either assumption for a few values of $k$ :
$k=1.0$
0.5
0.3
0.2
0.9866
0.1
0.0
$\kappa(\mathrm{I})=0.5000$
0.9125
0.9695
0.8333
0.9967
1.0000
$\kappa($ II $)=0.5000$
0.6667
0.7692
0.9091
1.0000

(The remainder of p. 175 is unaffected.) On the sine-law the total range of inclinations $\theta^{\prime}$ in the dry film is $0^{\circ}$ to $a=\arcsin k$, and on the tangent-law, $0^{\circ}$ to $90^{\circ}$ (as before).

Finally, concerning the distribution through these angle ranges (p. 176), if $N^{\prime} d \theta^{\prime}$ be the number of grains whose inclinations in the final state fall within the interval $\theta^{\prime}$ to $\theta^{\prime}+\mathrm{d} \theta^{\prime}$, we have $N^{\prime} \mathrm{d} \theta^{\prime}=N \sin \theta \mathrm{d} \theta$.

Thus $\left(\frac{\mathrm{d} \theta}{\mathrm{d} \theta}\right.$, being as on p. 176$)$ we have, on the sine-law, the final distribution law

$$
N^{\prime} \mathrm{d} \theta^{\prime}=N \frac{\cos \theta^{\prime} \sin \theta^{\prime} \mathrm{d} \theta^{\prime}}{k \sqrt{k^{2}-\sin ^{2} \theta^{\prime}}}, \quad 0<\theta^{\prime}<\arcsin k
$$


and, on the tangent-law,

$$
N^{\prime} \mathrm{d} \theta^{\prime}=N \frac{\sin \theta^{\prime} \mathrm{d} \theta^{\prime}}{k^{2} \cos ^{2} \theta^{\prime}+\sin ^{2} \theta^{\prime \prime}}, \quad 0<\theta^{\prime}<\frac{\pi}{2} .
$$

Formula $\left(4^{s}\right)$ gives, for the number $n^{\prime}\left(\theta^{\prime}\right)$ of grains whose inclinations fall within the interval 0 to $\theta^{\prime}$,

where $u=\sin ^{2} \theta^{\prime}$, i.e.,

$$
\mathrm{n}^{\prime}\left(\theta^{\prime}\right)=\int_{0}^{\theta^{\prime}} \mathrm{N}^{\prime} \mathrm{d} \theta^{\prime}=\frac{\mathrm{N}}{2 k} \int_{0}^{u}-\frac{\mathrm{d} u}{\sqrt{k^{2}-u}},
$$

$$
\mathrm{n}^{\prime}\left(\theta^{\prime}\right)=\mathrm{N}\left[1-\sqrt{1-\frac{\sin ^{2} \theta^{\prime}}{k^{2}}}\right]=\mathrm{N}[1-\cos \theta], \quad 0<\theta^{\prime}<a
$$

as it should be, for all these grains belonged originally to the range 0 to $\theta=\arcsin \left(\frac{\sin \theta^{\prime}}{k}\right)$. Similarly, in the case of the tangentlaw,

$$
\mathrm{n}^{\prime}\left(\theta^{\prime}\right)=\mathrm{N}[1-\cos \theta], \quad \theta=\arctan \left(\frac{\tan \theta^{\prime}}{k}\right), \ldots \ldots \ldots \ldots \ldots \ldots
$$

valid for any $\theta^{\prime}$ between 0 and $\frac{\pi}{2}$.

By $\left(5^{s}\right)$ we have, for example, for $\sin \theta^{\prime}=\frac{1}{2} k$,

$$
\mathrm{n}^{\prime}=\mathrm{N}\left(1-\frac{\sqrt{3}}{2}\right),
$$

i.e., 13.4 per cent of all grains (the remaining 86.6 per cent being contained between $\arcsin \frac{k}{2}$ and $\left.\arcsin k\right)$. For small $k$ formula $\left(5^{s}\right)$ can be written, approximately,

$$
\mathrm{n}^{\prime}\left(\theta^{\prime}\right)=\frac{1}{2} \mathrm{~N}\left(\frac{\theta^{\prime}}{k}\right)^{2} .
$$

The tangent-law formula $\left(5^{t}\right)$ gives for $\theta^{\prime}=45^{\circ}$, for instance, when $\tan \theta=1 / k$,

Thus, if $\dot{k}=\frac{1}{10}$,

$$
n^{\prime}\left(\frac{\pi}{4}\right)=N\left[1-\frac{k}{\sqrt{1+k^{2}}}\right] .
$$

$$
n^{\prime}\left(45^{\circ}\right)=0.905 N,
$$

that is to say, $901 / 2$ per cent of the grains have inclinations contained between $0^{\circ}$ and $45^{\circ}$, and the remaining $91 / 2$ per cent between $45^{\circ}$ and $90^{\circ}$.

EASTMAN KODAK Co

ROCHESTER, N. Y 